\title{
IdeAs
}

Idées d'Amériques

$11 \mid 2018$

Modernités dans les Amériques : des avant-gardes à aujourd'hui

\section{Arts visuels et stridentisme dans la revue mexicaine Irradiador (1923)}

Artes visuales y estridentismo en la revista mexicana Irradiador (1923)

Visual arts and stridentism in the Mexican review Irradiador (1923)

Isabelle Pouzet

\section{OpenEdition}

Journals

Édition électronique

URL : https://journals.openedition.org/ideas/2799

DOI : 10.4000/ideas.2799

ISSN : 1950-5701

Éditeur

Institut des Amériques

\section{Référence électronique}

Isabelle Pouzet, «Arts visuels et stridentisme dans la revue mexicaine Irradiador (1923) », IdeAs [En

ligne], 11 | 2018, mis en ligne le 24 juin 2018, consulté le 18 octobre 2022. URL : http://

journals.openedition.org/ideas/2799; DOI : https://doi.org/10.4000/ideas.2799

Ce document a été généré automatiquement le 18 octobre 2022.

\section{(i) $\ominus$

Creative Commons - Attribution - Pas d'Utilisation Commerciale - Pas de Modification 4.0 International - CC BY-NC-ND 4.0

https://creativecommons.org/licenses/by-nc-nd/4.0/ 


\title{
Arts visuels et stridentisme dans la revue mexicaine Irradiador (1923)
}

\author{
Artes visuales y estridentismo en la revista mexicana Irradiador (1923) \\ Visual arts and stridentism in the Mexican review Irradiador (1923)
}

\author{
Isabelle Pouzet
}

1 Qu'ils soient européens, comme le futurisme italien et l'ultraïsme espagnol, ou latinoaméricains, comme le créationnisme du Chilien Vicente Huidobro, les mouvements d'avant-garde du début du $\mathrm{XX}^{\mathrm{e}}$ siècle sont connus des intellectuels mexicains, dans les années 1920, grâce à des revues telles que Revista de revistas, Zig-Zag ou El Universal ilustrado qui s'en font le vecteur de diffusion (Schneider L. M., 1970 : 21). C'est dans ce contexte d'effervescence culturelle que le stridentisme fait son apparition sur la scène culturelle mexicaine sous l'impulsion de Manuel Maples Arce. Originaire de l'État de Veracruz, ce jeune poète vit à Mexico et publie ses textes de loin en loin dans la revue Revista de revistas. En 1921, il rédige le manifeste stridentiste qu'il intitule Actual $n^{\circ} 1$ et auquel il ajoute un sous-titre sans équivoque: Hoja de vanguardia. Comprimido estridentista. Son auteur décide de placarder cette feuille volante une nuit de décembre sur les murs de Mexico, dans le quartier des facultés. Il le fait également parvenir à des journaux mexicains, créant ainsi un vent de panique parmi les intellectuels et, en particulier, chez les membres de la Academia de la Lengua qui, craignant un attentat, ont demandé que leur bâtiment soit surveillé le lendemain soir (List Arzubide G., 1967 : 16-17).

2 Le stridentisme est d'abord un mouvement qui a pour vocation de renouveler la littérature telle qu'elle se pratique à cette époque au Mexique. En 1921, lorsqu'éclate le « feu d'artifice verbal » du stridentisme ${ }^{1}$, les lettrés de l'époque cultivent encore le goût pour le modernisme entretenu par le groupe Ateneo de la Juventud, dont le chef de file, José Vasconcelos, domine la scène littéraire mexicaine dès 1908. Un an après la naissance du stridentisme paraît la revue La Falange (1922), fondée par Jaime Torres Bodet et Bernardo Ortiz de Montellano, qui marche sur les traces de l'Ateneo et ne reconnaît pas encore les mouvements d'avant-garde européens (Escalante E., 1998). Le manifeste stridentiste vient donc jeter un pavé dans la mare. Germán List Arzubide, qui 
a tôt fait de rejoindre Manuel Maples Arce dans son aventure, se souvient des réactions qu'a provoquées le manifeste :

Verdadero manifiesto protestativo y desquiciador, sembró el espanto entre los gallineros líricos que se reunían cada semana para recitar versos azules y al final cambiarse las necesarias alabanzas. Los diarios y revistas atacaron con furia en que se adivinaba más que otra cosa, la amargura de tener que confesar ante el público que se ignoraba, con una ignorancia de cocinera, qué cosa era la nueva literatura (cité in Schneider L. M., $1970: 42)^{2}$.

3 Dans son autobiographie Soberana juventud, publiée quarante ans plus tard, Manuel Maples Arce rappelle que l'objectif de son manifeste était de "torcerle el cuello al Doctor González Martínez » (Maples Arce M., 2010: 84)³, c'est-à-dire de renverser la poésie en vogue du poète Enrique González Martínez.

4 À l'instar des mouvements d'avant-garde européens, le stridentisme se définit essentiellement par ce qu'il rejette et s'avère moins catégorique à propos de ce qu'il revendique. Dans le second numéro d'Actual, le poète veut faire table rase du passé et dit adorer le progrès. Il critique l'art bourgeois et lui préfère les objets modernes. Par exemple, parmi les différents points développés dans la revue, Manuel Maples Arce met symboliquement Chopin sur la chaise électrique (Schneider L. M., 1970 : 38). Manuel Maples Arce affirme, dans son premier manifeste, qu'il faut écrire de la «[...] poesía pura, suprimiendo todo elemento extraño y desnaturalizado (descripción, anécdota, perspectiva)»(ibid., p. 40) ${ }^{4}$. Ce précepte vaut aussi pour l'art en général qui doit se libérer de tout ornement inutile.

$\mathrm{Si}$, au départ, le poète est seul dans sa démarche, il va rapidement être rejoint par d'autres artistes comme le journaliste et auteur de chroniques Germán List Arzubide, le peintre Fermín Revueltas et l'écrivain Arqueles Vela. En 1922, Manuel Maples Arce publie son recueil Andamios interiores et Arqueles Vela, la Señorita etcétera. Par ailleurs, en 1923, lorsque paraît le premier numéro de la revue Irradiador, le stridentisme constitue un mouvement dont les créations artistiques vont de la poésie à la peinture, en passant par la photographie. L'objectif de Manuel Maples Arce n'est pas pour autant de fonder une école, ni d'imposer une ligne de conduite à qui que ce soit mais, comme l'affirme Arqueles Vela dans le deuxième numéro de la revue Irradiador, le stridentisme est pour lui « [...] un gesto. Una irrupción contra el reaccionarismo intelectual » (Vela A., 1923, s. p.) $)^{5}$.

Le stridentisme a laissé des traces écrites qui ont été abondamment étudiées par la critique. En revanche, celle-ci s'est peu attardée sur les dessins, les peintures et les gravures que certains artistes tels que Jean Charlot, Rafael Sala, Ramón Alva de la Canal, Fermín Revueltas ou encore Leopoldo Méndez ont réalisés en participant à l'aventure stridentiste. La récente édition en fac-similé de la revue Irradiador de Manuel Maples Arce va donc nous donner l'occasion d'aborder ce mouvement d'avant-garde par un autre biais, celui des arts visuels.

\section{Vers une redécouverte du stridentisme}

7 À la fin de l'année 1923, Manuel Maples Arce et Fermín Revueltas fondent la revue Irradiador. Le premier numéro paraît en septembre, le second numéro en octobre et le troisième et dernier numéro en novembre. Pendant plusieurs décennies, ces trois numéros sont restés introuvables et le chercheur mexicain Luis Mario Schneider - l'un 
des premiers à travailler sur ce mouvement - a toujours déploré la perte de cette revue dont il n'a jamais retrouvé la trace. C'est le chercheur et professeur de l'Université Autonome Métropolitaine d'Iztapalapa, Evodio Escalante, qui, par un heureux hasard, a découvert un exemplaire de cette revue ; il est à l'initiative de sa publication par l'UAM et en fac-similé, en 2012, sous le titre: Irradiador. Revista de vanguardia (Escalante E., $2012: 11-44)$.

Cette édition constitue un véritable joyau, tant par la rareté de la revue que par la qualité de l'édition qui a conservé la mise en page et les couleurs d'origine. À la différence des éditions en fac-similé des revues mexicaines $\mathrm{du} X \mathrm{XX}^{\mathrm{e}}$ siècle qu'a réalisées le Fondo de Cultura Económica, qui étaient en noir et blanc, les nombreuses illustrations de cette édition sont reproduites à l'identique et en grand format. Ce détail est important et il nous amène à nous intéresser aux images que renferme cette publication. En effet, le stridentisme a surtout été abordé à travers ses textes, qu'ils prennent la forme de poèmes comme ceux de Manuel Maples Arce et de Germán List Arzubide, ou de chroniques comme celles d'Arqueles Vela. Dans l'introduction à la revue, Serge Fauchereau et Evodio Escalante s'attardent davantage sur les écrits contenus dans Irradiador que sur les images qui s'y trouvent. Serait-ce que celles-ci revêtent moins d'importance que les textes, poèmes et autres articles qu'héberge la revue ? Ces images doivent-elles être considérées comme des illustrations ou bien comme des œuvres à part entière? Afin de répondre à ces interrogations, nous nous attarderons sur les images présentes dans la revue en nous efforçant de les interpréter à la lumière des idées qu'elle y sème.

9 Fruit de la collaboration entre un poète et un peintre, la revue Irradiador n'a pas été pensée comme une revue littéraire mais comme une revue d'art. Le néologisme employé pour son titre provient du verbe irradiar et semble suggérer que le stridentisme a pour vocation de se propager en brillant en même temps qu'il réchauffe. D'après Evodio Escalante, ce terme à la consonance scientifique aurait peut-être été inspiré par le titre d'un recueil de poèmes d'un auteur catalan (ibid., p. 19). Le sous-titre qui l'accompagne : «Revista de vanguardia. Proyector internacional de nueva estética publicado bajo la dirección de Manuel Maples Arce y Fermín Revueltas ${ }^{6}$ est, quant à lui, pensé dans une perspective qui porte sur l'art en général: la revue est conçue comme un "projecteur international », autrement dit, comme l'instrument optique servant à éclairer l'image statique ou mobile d'une "nouvelle esthétique ». Dès le départ, les termes qui définissent la revue font la part belle aux arts visuels et promettent de nous en proposer une vision à la fois internationale et inédite.

10 L'allure de la revue a été elle aussi conçue dans ce sens. Observons la couverture : le titre et le numéro de la revue se déclinent en trois couleurs différentes selon les parutions (vert pour le premier numéro, orange pour le deuxième et bleu pour le troisième) et, bien que le titre à la typographie très géométrique coiffe l'ensemble, c'est davantage le numéro de la revue, dont le coloris rappelle celui du titre qui, d'emblée, attire le regard. Une illustration se trouve à droite de chaque première page : dans le premier numéro, il s'agit d'une peinture de Fermín Revueltas, dans le deuxième, d'une peinture de Diego Rivera et, dans le dernier, d'une photographie d'Edward Weston. Enfin, la quatrième de couverture met elle aussi l'art au premier plan à travers une illustration d'inspiration cubiste, où les mots se heurtent les uns aux autres, comme happés par des demi-cercles et des diagonales. Dans un cadre de la même couleur que la 
page de titre, l'art visuel et les mots fusionnent pour donner un résultat paradoxalement chaotique et harmonieux.

Figure 1 : Couverture de Irradiador $\mathrm{n}^{\circ} 2$ (octobre 1923) // Figure 2 : Quatrième de couverture de Irradiador $\mathrm{n}^{\circ} 2$ (octobre 1923)

(c) Evodio Escalante

\section{IRRADIADIR}

REVISTA DE VANGUARDIA. PROYECTOR INTERNACIONALDE NUEVAESTETICA PUBLICADO BAJOLADIRECCION DE MANUEL MAPLES ARCE \& FERMIN REVUELTAS.

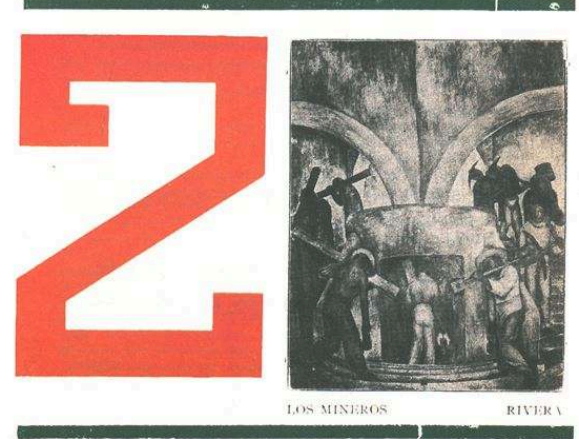

DEPOSITO GENERAL: - LIBRERIA DE CESAR CICERON. - AV, MADERO 56. - MEXICO, D. F.
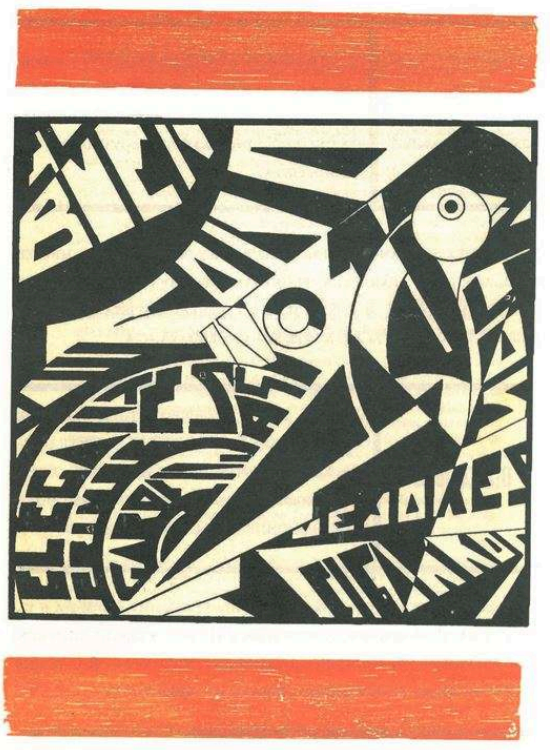

Dans cette revue, les images revêtent trois fonctions distinctes: il y a tout d'abord celles qui entrent en résonance avec les théories stridentistes tout en s'en faisant les vecteurs, puis celles qui tentent d'appliquer visuellement les préceptes stridentistes, et enfin celles qui surgissent au détour de la revue sans présenter de lien apparent avec les textes qu'elles accompagnent et qui semblent se suffire à elles-mêmes.

\section{Irradiador : un remède stridentiste}

12 Le premier numéro de la revue s'ouvre sur un texte aux allures de manifeste s'intitulant « Irradiación inaugural $»^{7}$ et, bien qu'il soit anonyme, on devine que Manuel Maples Arce en est l'auteur. Celui-ci s'adresse directement au lecteur qu'il agresse et loue en même temps en ces termes : «[...] quizá es Ud. todavía un imbécil; Ud. tiene talento. Ahora se ha extraviado Ud. en los pasillos vacíos de su imaginación» (Irradiador, 1923: s. p.) ${ }^{8}$. La langue est volontairement obscure et se construit au fil des lignes par des associations de mots qui sont si nombreuses qu'elles nuisent volontairement au sens. À la page suivante, le lecteur va trouver de quoi calmer les angoisses qu'a fait naître en lui ce discours désordonné et incohérent. Le remède stridentiste est là et en voici l'ordonnance :

Figure 3 : Calligramme stridentiste dans Irradiador $n^{\circ} 1$ (septembre 1923)

(c) Evodio Escalante 


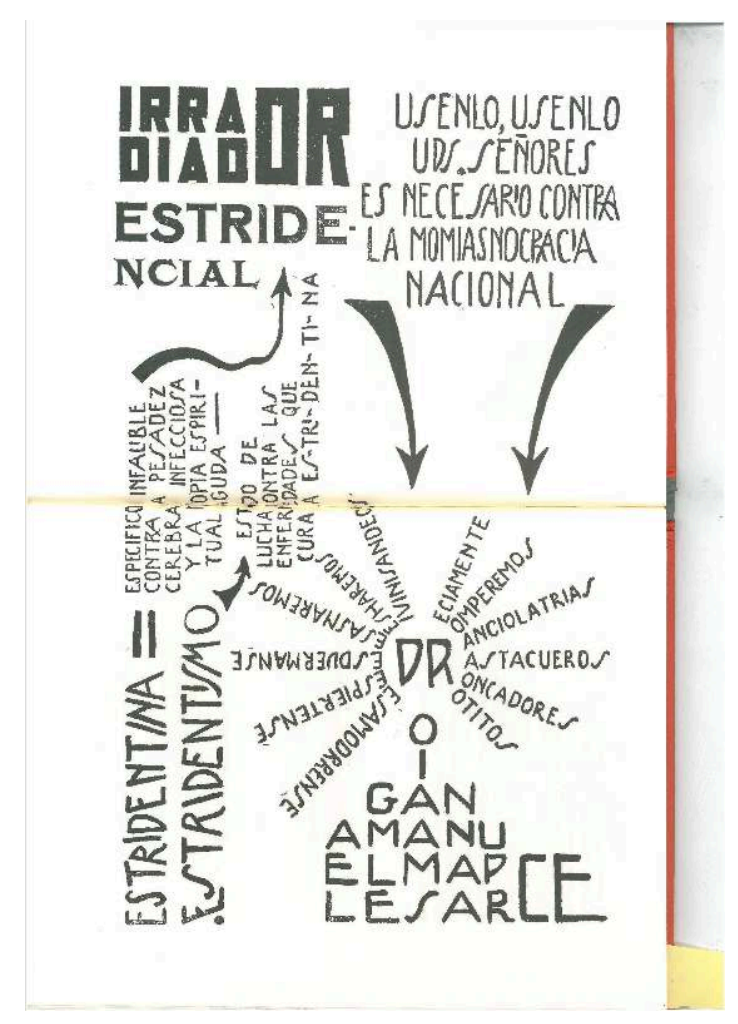

Dans ses mémoires publiées en 1967 sous le titre Soberana juventud, Manuel Maples Arce explique que ce calligramme a été imaginé au coin d'une table, au dos d'un menu, par lui-même, Diego Rivera et l'écrivain Julio Torri (Maples Arce M., 2010: 128). Le calligramme se présente comme une publicité vantant les propriétés d'un remède magique, la stridentine, qui, nous dit-il, est infaillible contre la "pesadez cerebral infectiosa» et la "miopía espiritual aguda» et qui aide à lutter contre la «momiasnocracia nacional» (Irradiador, 1923: s. p.) $)^{9}$. Un soleil au centre duquel brillent les initiales DR de Diego Rivera, qui sont aussi celles de "Doctor", irradie les lecteurs par ses rayons qui les enjoignent à sortir de leur léthargie tout en les invectivant et en les traitant de "rastacueros ", de "roncadores » et de "rotitos » $(\text { ibid. })^{10}$. Rien de tel donc qu'une bonne dose de stridentine pour sortir le lecteur de la torpeur culturelle et spirituelle dans laquelle il semble plongé. Ce calligramme, qui rappelle la rhétorique des mouvements d'avant-garde européens tels les ultraïstes en Espagne, présentant leur mouvement comme le remède à un grand nombre de maux, apparaît donc au service des préceptes stridentistes, à l'instar du manifeste Actual ou même du petit manifeste "Irradiación inaugural " qui ouvre le premier numéro. Néanmoins, ce calligramme est la seule image dans les trois numéros de la revue qui cherche à persuader le lecteur du bien-fondé du stridentisme. En effet, les représentations visuelles qui s'efforcent de mettre en image les théories stridentistes et de les illustrer y sont plus nombreuses.

\section{La mise en images des mots stridentistes}

Dans Actual, Manuel Maples Arce avait bien signifié qu'il ne tentait pas de fonder une école mais simplement de dépouiller les arts de leurs ornements inutiles pour revenir à un mode d'expression plus sincère. Dans le second numéro d'Irradiador, l'écrivain 
guatémaltèque Arqueles Vela consacre un article entier au stridentisme qu'il intitule "El estridentismo y la teoría abstraccionista " ${ }^{11}$ et qu'il présente dans une langue limpide, sans néologisme ni invective au lecteur. Il insiste sur le caractère ouvert du mouvement qui s'efforce d'atteindre les émotions et de les traduire simplement, sans artifice :

Toda esa literatura está basada en una ecuanimidad que no tiene la vida. Lo real y lo natural en la vida es absurdo. Lo inconexo. Nadie siente ni piensa en una perfecta continuidad. Nadie vive una vida como la de los personajes de las novelas románticas. Nuestra vida es arbitraria y los cerebros están llenos de pensamientos incongruentes (Vela A., 1923 : s. p.) ${ }^{12}$.

C'est justement cette fragmentation de l'esprit invoquée par Arqueles Vela que l'on trouve représentée au gré des pages d'Irradiador. La première illustration est un calligramme de Gonzalo Deza Méndez, reproduit sur une double page dans le deuxième numéro et qui porte le titre de La marimba en el patio. Sur la première partie du calligramme, les mots reproduisant visuellement les bruits de la cour commune de la vecindad s'étendent verticalement. En lisant de gauche à droite, la fréquence des bruits habituels s'accentue - "gritos de muchachos/ ladrar de perros" (Deza Méndez G., 1923 : s. p. $)^{13}$ - pour devenir «ladridos/ gritos/ ladridos » (ibid. $)^{14}$ et crée un désordre phonique qui contraste avec la tranquillité du chat qui dort et des vêtements qui sèchent au soleil. Mais l'organisation de la première page laisse place à un autre temps et à une autre musique : il pleut et les gouttes représentées par des traits en diagonale annoncent les notes de la marimba qui se dévoilent lettre par lettre, ou plutôt note après note, et qui font cesser le brouhaha ambiant. Ce calligramme se présente donc comme une tentative d'application visuelle des idées stridentistes : tant par sa position sur la page que par son sens, chaque mot a son importance dans l'histoire que livre son auteur.

S'il était aisé pour le lecteur de la revue de saisir le sens du calligramme de Gonzalo Deza Méndez, il n'en va pas de même pour l'œuvre d'un autre artiste, Pedro Echeverría: signée de son pseudonyme Polo-As, "Solsticios » est présentée sur une double page du dernier numéro. Le poème prend ici la forme d'une partition sur laquelle s'égrènent des lettres formant des mots dont seuls certains sont compréhensibles. Il se crée alors un contraste entre les lignes parfaites et les lettres qui semblent s'enchevêtrer de manière aléatoire, rendant ainsi l'œuvre difficile à comprendre. Aussi « Solsticios » est-il l'exacte illustration du petit manifeste de Manuel Maples Arce puisqu'il met en lumière ce qu'Evodio Escalante appelle les «jeroglíficos estridentistas $»^{15}$ (Escalante E., $2012: 26$ ).

Parmi les images de la revue que l'on peut qualifier de «stridentistes ", il y a celles qui ont pour fonction d'accompagner le texte en l'illustrant. Le thème choisi est la ville, ses formes aiguisées en traduisent la cacophonie. Malgré la mauvaise qualité de sa reproduction, le dessin intitulé El restorán que Fermín Revueltas a proposé pour la couverture du numéro 1 d'Irradiador, dépeint lui aussi un univers urbain avec, au premier plan, la table d'un restaurant et au second plan, la ville dont le mouvement incessant est suggéré par les différentes lignes de fuite qui s'entremêlent au point de se confondre.

18 Enfin, Fermín Revueltas a choisi de proposer le même dessin pour la quatrième de couverture des deux premiers numéros. Il est important de préciser ce détail car il montre à quel point la revue est soignée. Il souligne la volonté de ses fondateurs de 
laisser une dernière impression "stridentiste" au lecteur. Cependant, au troisième numéro, à la différence des deux premières parutions, l'image sur laquelle se clôt la revue est une illustration publicitaire. Cette fois encore, le choix de l'image est révélateur de cette volonté de cohérence entre le contenu de la revue et son apparence. Il s'agit d'une publicité d'inspiration cubiste pour les cigarettes $E l$ Buen Tono qui est remarquable pour l'esprit qu'elle dégage. Ce n'est pas la première fois que les stridentistes font référence aux cigarettes de cette marque : elles faisaient déjà partie du décor urbain peint par Manuel Maples Arce au paragraphe VI de son manifeste Actual $n^{\circ} 1$. Au même titre que la brasserie Moctezuma de Orizaba ou que le garage Haynes cités dans sa description hallucinée de la capitale, les cigarettes El Buen Tono constituent un symbole de la modernité urbaine :

Accesorios de automóviles, refacciones Haynes, llantas, acumuladores y dinamos, chasis, neumáticos, Klaxons, bujías, lubricantes, gasolina. Estoy equivocado. Moctezuma de Orizaba es la mejor cervecería en México, fumen cigarrillos del Buen

Tono, S. A., etc., etc. ${ }^{16}$.

Tout comme dans le dessin de Fermín Revueltas, les formes sont ici très géométriques : les ovales rappelant les ronds de fumée émis par le personnage de gauche se mêlent à des demi-cercles représentant des bâtiments aux allures de soucoupes volantes. La vision nocturne de la ville est chaotique : les pancartes publicitaires clignotent sans cesse, les lumières de la ville fonctionnent à plein régime, les câbles téléphoniques strient le ciel étoilé et les rues se croisent dans un dédale d'arcs de cercle qui semblent ne mener qu'à l'éclat radiophonique des cigarettes.

Figure 4 : Publicité El Buen Tono dans Irradiador $n^{\circ} 3$ (novembre 1923)

(c) Evodio Escalante

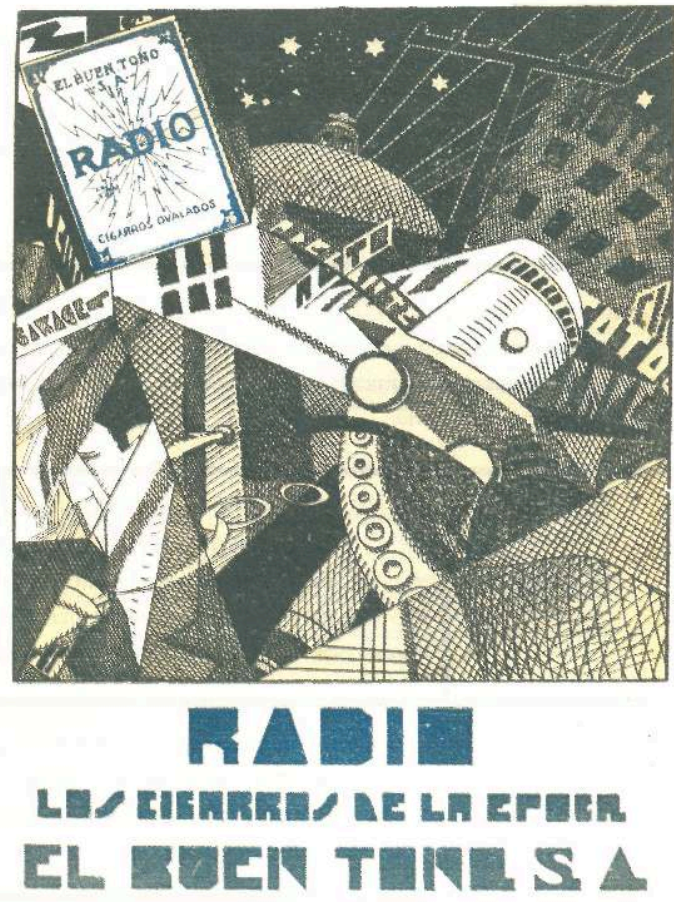




\section{Les œuvres autonomes}

Weston figurant sur la page de couverture du troisième numéro d'Irradiador. Intitulée Steel, elle a été prise en octobre 1922 à l'usine métallurgique Armco Company de Middleton dans l'ohio et donne à voir une rangée de cheminées d'usine en contreplongée (Fauchereau S., 2012 : 53). Le photographe nord-américain a vécu un peu plus d'une année au Mexique où il a fait la connaissance des stridentistes qui lui ont donné l'opportunité, le 12 avril 1924, d'exposer ses œuvres au Café de Nadie - le café des stridentistes à Mexico -, aux côtés de celles de Jean Charlot, Rafael Sala, Ramón Alva de la Canal, Fermín Revueltas, Leopoldo Méndez entre autres, tandis que les poètes Manuel Maples Arce et Germán List Arzubide lisaient leurs poèmes. Sa collaboration ne s'arrête pas là car l'une de ses photographies va illustrer le livre de List Arzubide El movimiento estridentista publié en 1926, et une autre sera utilisée en première page de la revue Forma en 1928 (Conger A., 1983: 18). Ce premier séjour au Mexique a été déterminant dans la carrière d'Edward Weston, point tant pour le succès qu'il y a rencontré que pour l'impact que la culture, les paysages et l'artisanat mexicains ont eu sur sa propre activité. Dans son introduction à l'édition en fac-similé d'Irradiador, Serge Fauchereau explique qu'il s'agissait de l'unique photographie qu'il avait rapportée des États-Unis et qu'il l'appréciait beaucoup, ce qui peut expliquer sa présence au sein de la revue (Fauchereau S., 2012 : 53). Au même titre que la peinture de Fermín Revueltas du premier numéro, cette photographie traduit l'esprit stridentiste, non pas cette fois grâce à un paysage urbain mais grâce à un décor industriel. La modernité est suggérée par un motif récurrent dans l'esthétique stridentiste : d'immenses cheminées d'usine se dressent dans le ciel obscurci par les fumées qui s'en échappent ${ }^{17}$

Parmi les œuvres que publie Irradiador, certaines semblent loin des préoccupations stridentistes : il s'agit de trois gravures de Jean Charlot, d'une peinture de Diego Rivera et d'un dessin de Leopoldo Méndez. Avant d'analyser ces images, il convient de revenir sur les liens qui unissent ces trois artistes au fondateur du stridentisme.

Le peintre français Jean Charlot est arrivé en 1921 au Mexique, plus exactement à Mexico où il a fait la connaissance des stridentistes qu'il a suivis tout au long de leur aventure et auxquels il a été fidèle. Ses œuvres ont fortement marqué les jeunes muralistes que sont à l'époque Diego Rivera et David Alfaro Siqueiros. Quant à Leopoldo Méndez, ami de Manuel Maples Arce depuis l'adolescence, il a été le premier artiste à se lancer dans l'aventure stridentiste (Reyes Palma F., 1994: 11). Par la suite, il accompagne les stridentistes à Xalapa, ville qu'ils ont rebaptisée Estridentópolis et collabore à la revue Horizonte qu'ils publient à partir d'avril 1926. Leopoldo Méndez va enseigner le dessin dans les écoles et, dès 1929, il le fait en tant qu'instituteur missionnaire (ibid., p. 8-9).

24 La reproduction de la fresque de Diego Rivera Entrada a la mina (1923), qui orne actuellement le bâtiment du Ministère de l'Education (SEP), vient illustrer la première page du deuxième numéro de la revue sous le titre Los mineros. Ici les mineurs 
s'apprêtent à descendre dans la mine en cadence, leurs outils sur l'épaule, les visages dans l'ombre ou bien cachés par un chapeau dans un mouvement circulaire, symbole de leur travail incessant. Cette absence de caractéristiques propres ainsi que la composition même de la scène traduisent toute la difficulté de leur tâche en même temps qu'elles la dénoncent.

On retrouve ce regard plein de compassion pour les travailleurs dans les trois gravures que Jean Charlot a proposées pour Irradiador. Les deux premières paraissent dans le premier numéro et la troisième le mois suivant. Toutes les trois sont des gravures sur bois mettant en scène un Mexicain au travail. Les deux gravures publiées dans le premier numéro d'Irradiador soulignent la dureté de la vie paysanne mexicaine : la première représente un paysan ou un commerçant à l'échine courbée sous le poids des animaux qu'il transporte et la deuxième une paysanne portant son enfant sur son dos. Dans la dernière gravure, une paysanne est en train de cuisiner à même le sol.

Tout comme Jean Charlot, l'artiste mexicain Leopoldo Méndez met en scène la vie des travailleurs dans le dessin à l'esprit cubiste intitulé La costurera, qu'il publie dans le troisième et dernier numéro de la revue. À l'époque, il ne pratique pas encore la gravure mais seulement le dessin, et celui-ci est l'un des premiers qu'il publie. C'est à nouveau une scène de la vie quotidienne paysanne que l'artiste nous livre ici: une femme seule, penchée sur son ouvrage, s'attelle à un travail de couture que l'on imagine lent et minutieux. La douceur de la scène est suggérée par la lumière qui inonde la pièce de ses rayons arrondis et par la présence d'un couffin où l'on devine un enfant endormi. De même que dans les gravures de Jean Charlot et dans le dessin de Diego Rivera, le visage de la protagoniste est volontairement effacé, celle-ci étant dessinée par des traits grossiers qui ne laissent percevoir que sa silhouette.

Figure 5 : La costurera de Leopoldo Méndez dans Irradiador n 3 (novembre 1923) 


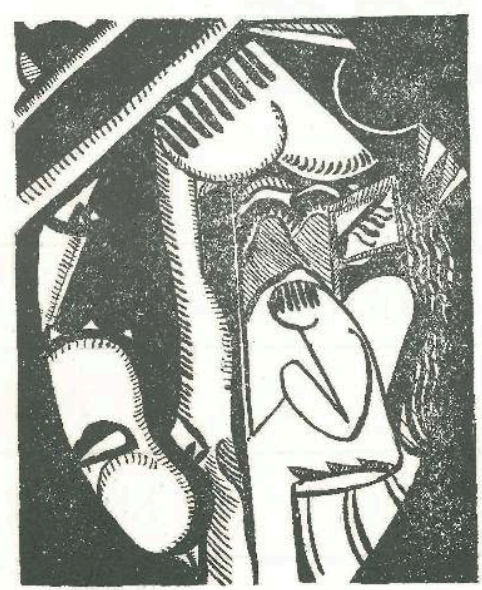

DAE COBTURERA. DIBUJO traitant de l'exploitation du pétrole au Mexique et un autre portant sur la culture précolombienne ${ }^{18}$ - prouvent bien que le stridentisme n'a pas seulement consisté en une explosion verbale consacrée à la ville. Dans son ouvrage Fundamentos de la literatura mexicana publié plusieurs décennies plus tard, Arqueles Vela a insisté sur le caractère social de ce mouvement artistique : "Por primera vez en la poesía mexicana, la individualidad desmesurada encuentra una resonancia social» (Vela A., 1966: 129) ${ }^{19}$. Aussi, Irradiador, tout en se faisant le vecteur de diffusion du stridentisme, vient-il à sa manière révéler certaines problématiques sociales (voir Mora F. J., 2000 et Rashkin Elissa J., 2012).

Conclusion

Si la fin de l'aventure Irradiador ne signe pas la fin de l'aventure stridentiste, la durée de vie de la revue a pourtant été à l'image du mouvement lui-même, bien courte. Pour des raisons obscures, Irradiador n'a plus paru après le troisième numéro alors qu'un quatrième numéro était en préparation (Maples Arce M., 2010 : 91). Toutefois, la revue Horizonte, qui sera publiée à Xalapa, lui succède dès 1926. Après Mexico, les stridentistes s'établissent à Xalapa; mais, lorsqu'en en 1927 le protecteur des stridentistes, le général Heriberto Jara, est démis de ses fonctions, le mouvement prend fin. 
30 Alors que la revue Irradiador est restée introuvable pendant des décennies, l'édition en fac-similé que propose Evodio Escalante donne l'occasion de jeter un regard nouveau sur ce mouvement d'avant-garde mexicain. Cette édition ne vient nullement contredire ou même interroger ce qui a été écrit ou ce qui a été dit sur le stridentisme, mais elle montre à quel point celui-ci n'est pas un mouvement uniquement littéraire mais bel et bien un mouvement qui s'exprime à travers les arts visuels. Les œuvres qui figurent dans cette revue et que nous avons commentées ne sont pas considérées comme des illustrations venant décorer ou même accompagner les textes stridentistes mais elles revêtent autant d'importance que ceux-ci. Leur position sur la page est d'ailleurs révélatrice du rôle que Manuel Maples Arce souhaite leur donner : elles sont isolées sur une page ou bien sur une double page et ne se trouvent en aucun cas sur la même page qu'un texte. En ce sens, Irradiador se distingue d'une revue qui lui est contemporaine, La Falange (1922-1923), fondée sous l'impulsion du Ministère de l'Education Publique (SEP), et où les dessins, les gravures et les peintures ne servent qu'à illustrer les textes de ses contributeurs. Il faudra attendre la revue Contemporáneos (1928-1931) pour que, tout comme dans Irradiador, les arts visuels soient mis sur le même plan que la littérature. En ce sens, l'initiative des fondateurs d'Irradiador est doublement avant-gardiste, car en même temps qu'elle fait souffler un vent d'idées nouvelles sur la scène culturelle mexicaine, elle révèle leur conception de la modernité qui englobe tous les arts.

\section{BIBLIOGRAPHIE}

Conger, Amy, Edward Weston in Mexico 1923-1926, Albuquerque, The University of New Mexico Press, 1983.

Deza Méndez Gonzalo, « La marimba en el patio », Irradiador, n², 1923.

Escalante, Evodio, « Los Proto-Contemporáneos en La Falange (1922-1923) », América. Polémiques et manifestes, n²1, 1998, p. 55-63.

Escalante, Evodio, « El descubrimiento de Irradiador. Nueva luz sobre el estridentismo » in Irradiador. Revista de vanguardia. édition fac-similé, collection « Espejos de la memoria », Iztapalapa, Universidad Autónoma Metropolitana, 2012, p. 11-44.

Fauchereau, Serge, «Irradiador en el espíritu de la época », in Irradiador. Revista de vanguardia. édition fac-similé, collection « Espejos de la memoria », Iztapalapa, Universidad Autónoma Metropolitana, 2012, p. 45-60.

Irradiador. Revista de vanguardia, édition fac-similé, collection « Espejos de la memoria », Iztapalapa, Universidad Autónoma Metropolitana, 2012.

List Arzubide, Germán, El movimiento estridentista, Mexico, Secretaría de Educación Pública, [1928] 1987.

Maples Arce, Manuel, Soberana juventud, Madrid, Editorial Plenitud, 1967.

Maples Arce, Manuel, Memorias I. Soberana juventud, Mexico, Universidad Veracruzana, 2010. 
Mora, Francisco Javier, « El estridentismo mexicano: señales de una revolución estética y política », Anales de Literatura Hispanoamericana, n²9, 2000, p. 257-275.

Prieto González, José Manuel, « El estridentismo mexicano y su construcción de la ciudad moderna a través de la poesía y la pintura ", Geo Crítica. Cuadernos críticos de Geografía Humana, vol. XVI, n³98, 2012, http://www.ub.edu/geocrit/sn/sn-398.htm\#_edn70, consulté le 24 avril 2018.

Schneider, Luis Mario, El estridentismo: Una literatura de la estrategia, Mexico, Instituto Nacional de Bellas Artes, 1970.

Rashkin, Elissa Joy, « La poesía estridentista : vanguardismo y compromiso social », Intersticios Sociales, $\mathrm{n}^{\circ} 4$, septembre- février 2012, p. 1-30.

Reyes Palma, Francisco, Leopoldo Méndez: El oficio de grabar, Mexico, Consejo Nacional para la Cultura y las Artes, 1994.

Vela Arqueles, « El estridentismo y la teoría abstraccionista », Irradiador, n², 1923.

Vela, Arqueles, Fundamentos de la literatura mexicana, Mexico, Ediciones Patria, 1966 [1953].

\section{NOTES}

1. Nous empruntons cette expression à Luis Mario Schneider qui parle de "pirotecnia verbal » pour désigner le stridentisme (Schneider L. M., $1970: 206$ ).

2. «Ce véritable manifeste protestataire et perturbant a semé la panique dans les poulaillers lyriques qui se réunissaient toutes les semaines pour réciter des vers fleuris et pour terminer en s'échangeant des compliments de circonstance. Dans les journaux et les revues qui ont répondu avec furie, on devinait l'amertume de devoir avouer au public leur méconnaissance de la nouvelle littérature. » Nous traduisons, de même que pour l'ensemble des citations de cet article.

3. «Tordre le cou au Docteur González Martínez». Manuel Maples Arce emprunte à Enrique González Martínez le premier vers de son célèbre sonnet «La muerte del cisne » : "Tuércele el cuello al cisne de engañoso plumaje ». Dans ce poème, le poète mexicain tente de rompre avec le modernisme hérité de Rubén Darío en cherchant à éliminer le cygne, symbole poétique du modernisme.

4. «De la poésie pure, en supprimant tout élément étranger et dénaturalisé (tel que la description, l'anecdote, la perspective. »

5. « [...] un geste. Une irruption de l'esprit contre le réactionarisme [sic] intellectuel. »

6. «Revue d'avant-garde. Projecteur international d'une nouvelle esthétique sous la direction de Manuel Maples Arce et de Fermín Revueltas ».

7. «Irradiation inaugurale».

8. «Peut-être que vous êtes encore un imbécile; vous avez du talent. Vous vous êtes perdu dans les couloirs vides de votre imagination ».

9. « Lourdeur cérébrale infectieuse ", « myopie spirituelle aigüe », « momieânocratie nationale ».

10. « Rastaquouères ", « ronfleurs " et « parvenus".

11. «Le stridentisme et la théorie abstractionniste».

12. «Toute cette littérature est basée sur une impartialité que la vie n'a pas. Ce qui est réel et naturel dans la vie, c'est l'absurde. L'incohérent. Personne ne sent ni ne pense avec une parfaite continuité. Personne ne vit une vie comme celle des personnages des romans romantiques. Notre vie est arbitraire et les cerveaux sont pleins de pensées incongrues. "

13. «Cris de garçons/ aboiements de chiens ». 
14. «Aboiements/ cris/ aboiements».

15. « Hiéroglyphes stridentistes ».

16. «Des accessoires pour automobiles, le garage Haynes, des jantes, des accumulateurs et des dynamos, des châssis, des pneumatiques, des klaxons, des bougies, des lubrifiants, de l'essence. Je me trompe. Moctezuma de Orizaba est la meilleure brasserie du Mexique, fumez des cigarettes $\mathrm{El}$ Buen Tono, S. A., etc., etc. " Le manifeste original est consultable en ligne sur le site de la Bibliothèque nationale de France: http://gallica.bnf.fr/ark:/12148/bpt6k899646s/f2.item, page consultée le 20 avril 2018.

17. José Manuel Prieto González montre que les usines et les fumées qu'elles dégagent, les gratteciels ou encore les poteaux électriques sont omniprésents dans l'esthétique stridentiste (Prieto González J. M., 2011).

18. Irradiador $n^{\circ} 2$, octobre 1923 : s. p.

19. «Pour la première fois dans la poésie mexicaine, l'individualité démesurée trouve un écho social».

\section{RÉSUMÉS}

Dans cet article nous nous proposons de revenir sur le stridentisme, le mouvement d'avant-garde créé par Manuel Maples Arce au début des années 1920 au Mexique. Son mouvement, qui n’a duré que quelques années, a été marqué par la parution de la revue Irradiador, fondée en collaboration avec le peintre muraliste mexicain Fermín Revueltas, en 1923. Tous ceux qui se sont intéressés au stridentisme ont déploré la perte de cette revue restée introuvable tout au long du $\mathrm{XX}^{\mathrm{e}}$ siècle. C'est le chercheur et professeur de l'Université Autonome Métropolitaine (UAM) d'Iztapalapa, Evodio Escalante, qui, par un heureux hasard, en a retrouvé un exemplaire; il est à l'initiative de sa publication en fac-similé en 2012 par l'UAM, sous le titre : Irradiador. Revista de vanguardia. Cette récente édition d'Irradiador va nous donner l'occasion de revenir sur le mouvement stridentiste à travers l'analyse des trois numéros qui la composent (septembre, octobre et novembre 1923). À la lumière des images publiées au fil des pages, nous nous efforcerons d'interroger le rapport à la modernité de cette revue qui, dès la page de titre, se présente comme le « projecteur international d'une nouvelle esthétique ».

Este artículo tiene como objeto de estudio el movimiento de vanguardia creado por Manuel Maples Arce a principios de los años 1920 en México: el estridentismo. A pesar de su corta vida, este movimiento fue marcado por la publicación de la revista Irradiador fundada en colaboración con el pintor muralista mexicano Fermín Revueltas, en 1923. Todos los que se interesaron en este movimiento siempre lamentaron la ausencia de esta revista que no se encontraba ni en las bibliotecas ni en los acervos especializados. Fue Evodio Escalante, profesor e investigador de la Universidad Autónoma Metropolitana (UAM) de Iztapalapa, quien encontró un ejemplar hace unos años. Este hallazgo afortunado lo llevó a publicar en 2012 una edición facsimilar de la revista que tituló: Irradiador. Revista de vanguardia. Esta reciente edición nos ofrece la ocasión de acercarnos al movimiento estridentista mediante el análisis de sus tres números (septiembre, octubre y noviembre de 1923). El estudio de las imágenes publicadas nos llevará a reflexionar acerca de la modernidad de esta revista que, desde la portada, se presenta como el "proyector internacional de una nueva estética". 
This article concerns Stridentism, the Mexican avant-garde movement created by Manuel Maples Arce at the beginning of the 1920s. Lasting only a few years, his movement was marked by the appearance of the Irradiador review, cofounded with the Mexican mural painter Fermin Revueltas in 1923. Those interested by Stridentism regretted that this review remained missing during the 20th century. The teacher and researcher Evodio Escalante found a copy by chance and published it in facsimile in 2012 at Iztapalapa University (UAM) under the title Irradiador. Revista de vanguardia. This recent edition of Irradiador gives us the occasion to return to the movement via the analysis of all three monthly issues -September, October and November 1923. In the light of the images published in its pages, we ask what is the relationship with modernity of this review which, from the title page onwards, presents itself as the «international projector of a new aesthetic ».

\section{INDEX}

Palabras claves : Irradiador, estridentismo, revistas, vanguardia, México

Keywords : Irradiador, stridentism, reviews, avant-garde, Mexico

Mots-clés : Irradiador, stridentisme, revues, avant-garde, Mexique

\section{AUTEUR}

\section{ISABELLE POUZET}

Isabelle Pouzet est Maître de conférences à l'Université du Littoral Côte d'Opale, elle est l'auteur d'une thèse portant sur la correspondance du poète mexicain Efraín Huerta et sur la genèse de son oeuvre de jeunesse. Elle s'intéresse actuellement aux manuscrits de poètes latinoaméricains (Carlos Pellicer, Raúl Zurita, Salvador Novo) et aux revues littéraires mexicaines de la première moitié $\mathrm{du} \mathrm{XX}^{\mathrm{e}}$ siècle 